\title{
PENSAMENTO COMPUTACIONAL E CÁLCULO DIFERENCIAL E INTEGRAL: PROPOSTA DE COMPONENTE CURRICULAR PARA $O$ IFMS - CAMPUS AQUIDAUANA
}

\author{
DOI: 10.37702/2175-957X.COBENGE.2021.3655
}

Maria Fernanda Romero da Silveira - maria.silveira@estudante.ifms.edu.br IFMS

Rua GIOVANE TOSCANO DE BRITO 2254

79200-000 - Aquidauana - MS

Vinícius Aparecido Reis de Andrade - coufinnette@gmail.com IFMS

Rua EXPEDICIONARIO CANDIDO GOMES 870

79210-000 - Anastácio - MS

Luan Matheus Moreira - luan.moreira@ifms.edu.br IFMS

Rua Giovane Toscano De Brito 1013

79200-000 - Aquidauana - MS

Resumo: No Curso Superior em Engenharia Civil do IFMS - Campus Aquidauana constatou-se uma elevada taxa de retenção principalmente nas unidades curriculares relacionadas ao Cálculo Diferencial e Integral. Após investigação na literatura observou-se que a utilização de estratégias de ensino-aprendizagem que colocam no estudante de forma ativa no processo tem gerado melhores indicadores de aprendizagem. Assim, o objetivo deste trabalho foi desenhar uma proposta de componente curricular focalizada aos estudantes que estão cursando a unidade curricular de Cálculo Diferencial e Integral I, respaldando-se a práxis pedagógica no Pensamento Computacional. A proposta foi desenvolvida e espera-se verificar sua efetividade em uma avaliação de impacto no futuro.

Palavras-chave: Letramento Computacional. Ensino-aprendizagem. Evasão. 


\section{PENSAMENTO COMPUTACIONAL E CÁLCULO DIFERENCIAL E INTEGRAL: PROPOSTA DE COMPONENTE CURRICULAR PARA O IFMS - CAMPUS AQUIDAUANA}

\section{INTRODUÇÃO}

Pensar computacionalmente aumenta o poder cognitivo e desenvolve a capacidade de resolução de problemas em todas as áreas do conhecimento, contribuindo de forma relevante à formação crítica. O ser humano deve parar de se preocupar com questões mecânicas e rotineiras que exijam velocidade, para passar à produção do conhecimento (BOSCARIOLI; KAMINSKI, 2019).

O pensamento computacional faz com que o estudante tenha que ordenar e analisar os dados por meio de diversas etapas que envolvem abstração, elaboração de modelos algoritmos e simulações, portanto estimula a capacidade crítica e analítica tanto da escrita quanto da linguagem matemática, desenvolvendo capacidades de planejamento, elaboração de estratégias, sequências e síntese (BOSCARIOLI; KAMINSKI, 2019).

Nos cursos de Engenharia costuma-se dispor, nos primeiros semestres, de unidades curriculares ligadas à Matemática, com destaque ao Cálculo Diferencial Integral (ou apenas Cálculo).

Conforme observa-se na literatura (e no IFMS - Campus Aquidauana), os níveis de retenção são consideravelmente elevados nos primeiros semestres dos cursos de Engenharia, o que incentiva a evasão por parte dos estudantes.

Assim, conceber estratégias para minimizar este fenômeno é uma ação de extrema importância para a permanência e o êxito dos estudantes.

De acordo com a Plataforma Nilo Peçanha (2019), a taxa de evasão no curso de graduação em Engenharia Civil, do IFMS - Campus Aquidauana, é de 12,8\% para o ano de 2019. E, de acordo com discussões realizadas com a Coordenação do Curso, as unidades curriculares relacionadas ao Cálculo Diferencial Integral estão na liderança em número de alunos reprovados.

Além disso, no Projeto Pedagógico de Curso em Engenharia Civil (IFMS, 2017), as unidades curriculares possuem pré-requisitos como instrumento de controle do processo de aprendizagem dos estudantes. Logo, quando o estudante reprova em Cálculo Diferencial e Integral I no $1^{\circ}$ semestre, ele não pode se matricular na unidade curricular de Cálculo Diferencial e Integral II ofertada no $2^{\circ}$ semestre. Sem cursar esta unidade, não poderá se matricular nas unidades de Cálculo Diferencial e Integral III do 3ํㅗ semestre e Mecânica Geral do $4^{\circ}$ semestre. Ou seja, uma reprovação em de Cálculo Diferencial e Integral I no $1^{\circ}$ Semestre tende a desencadear um processo emocional que poderá levar o estudante a evadir o curso.

Lobo (2012) indicou hipóteses causadoras da evasão no ensino superior, tais como: i) baixa qualidade da educação básica brasileira, ii) baixa eficiência do ensino médio, iii) limitação das políticas de financiamento do estudante, iv) escolha precoce da especialidade profissional, v) dificuldade de mobilidade estudantil, vi) rigidez do arcabouço legal e das exigências para autorização/reconhecimento de cursos, vii) falta de pressão para combater a evasão, viii) legislação sobre a inadimplência no Brasil e ix) enorme quantidade de professores despreparados para o ensino e para lidar com o estudante real. 
Soares (2006) realizou uma série de sugestões para minimizar a evasão de estudantes em cursos de Engenharia, com base nas características da Universidade Federal do Rio de Janeiro (UFRJ) na época em questão. Dentre as medidas, cabe destacar a necessidade de um Corpo de Professores atuante, onde os mesmos não apenas ministram aulas, mas também, tenham aptidão para "orientar os alunos academicamente".

Reis (2012) realizou uma investigação no Centro Federal de Educação Tecnológica do Rio de Janeiro (CEFET/RJ) e também identificou várias medidas para buscar a minimização da evasão nos cursos de engenharia, onde destacam-se: 1) a integração do conhecimento, por meio da união de disciplinas, atividades práticas e extracurriculares; 2) a mudança nas metodologias de ensino; e 3) a capacitação dos docentes a fim de preparálos para o desenvolvimento de projetos inovadores, com a constante revisão de suas atividades.

Barbosa (2004), indicou a deficiência de conhecimentos básicos de matemática advindos do ensino fundamental e médio como um dos principais elementos que geram externalidades negativas no processo de ensino e aprendizagem de Cálculo Diferencial e Integral. Também ressaltou que a utilização de métodos de ensino baseados na "transferência de conhecimentos" é ainda amplamente utilizada pelos professores.

Oliveira e Raad (2012) apresentaram a reprovação e o insucesso na disciplina de Cálculo como uma cultura escolar - que é fruto de pesquisa no cenário nacional e internacional - os autores destacaram que, mesmo com as mudanças didáticas de alguns professores e os esforços dos departamentos de Matemática das instituições de ensino superior em ofertar cursos intensivos e monitoria, não foi suficiente para ao menos minimizar os elevados índices de insucesso da disciplina no Brasil.

Santos (2018) identificou que a Educação Básica não cria condições para o pleno desenvolvimento dos estudantes em Matemática, de modo a torná-los aptos aos desafios do Ensino Superior em cursos de Ciências Exatas. O autor sugere que um curso de nivelamento baseado em estratégias pedagógicas mais atraentes aos desafios da atualidade pode ser um mecanismo de minimização da evasão.

Veloso, Couto e Valentim (2018) apresentaram evidências de que o nivelamento é um mecanismo importante para facilitar a inserção e motivar a permanência dos ingressantes. Em relação à instituição de ensino investigada, identificaram que as deficiências de aprendizagem em matemática e física foram mais acentuadas nos alunos que cursaram o Ensino Médio em escola pública.

De acordo com esta literatura, observou-se que uma das principais variáveis que explicam o fato social da evasão em Cálculo é o baixo nível de desenvolvimento de competências em Matemática provenientes do Ensino Médio e a utilização de métodos e estratégias de ensino baseados na transferência de conhecimentos ao invés de desenvolvimento de competências.

\section{$1.1 \quad$ Objetivos}

Desenvolver uma proposta de componente curricular focalizada aos estudantes que estão cursando a unidade curricular de Cálculo Diferencial e Integral I do Curso Superior em Engenharia Civil do IFMS - Campus Aquidauana, fundamentando-se na práxis pedagógica do Pensamento Computacional. 


\section{METODOLOGIA}

Esta pesquisa tem uma abordagem exploratória pois trata-se de uma investigação teórica fundamentada na literatura disponível e no problema identificado no IFMS - Campus Aquidauana.

Desta forma, as etapas para o desenvolvimento ocorreram conforme a seguir.

1. Investigação na literatura acadêmica sobre o que é o pensamento computacional e como pode ser utilizado como estratégia de ensino e aprendizagem.

2. Investigação nos projetos pedagógicos dos principais cursos de engenharia do país, nos quais são oferecidas unidades curriculares de Pré-Cálculo.

3. Organização dos objetivos de aprendizagem em Pré-Cálculo a partir da Taxonomia de Bloom.

4. Organização dos conteúdos programáticos em Pré-Cálculo a partir da investigação dos currículos de outras iniciativas.

\section{DESENVOLVIMENTO}

O Raciocínio Computacional envolve focar apenas naquilo que é realmente relevante de se solucionar, ou seja, situações irrelevantes devem ser ignoradas. Criando a representação do que se procura resolver ou passar informação (abstração).

Depois de identificar o problema, deve-se quebrá-lo em pequenos outros problemas - em partes menores (que são mais fáceis de se resolver) - o que favorece a compreensão de novas situações. A decomposição de problemas é o processo de identificar e utilizar fórmulas, funções e procedimentos matemáticos necessários para solucionar os problemas. Segundo Liukas (2015) a decomposição é um processo pelo qual os problemas são decompostos em partes menores.

Porém, cada um desses problemas menores pode ser analisado individualmente com maior profundidade, identificando soluções usadas em problemas passados que podem ser adaptadas para solucionar uma variedade de problemas similares. De acordo com Liukas (2015) "consiste em encontrar similaridades e padrões com o intuito de resolver problemas complexos de forma mais eficiente".

Por último, passos podem ser criados para resolver cada um dos subproblemas encontrados. Segundo Piaget (1990) "a aprendizagem é concebida como registro de dados exteriores". Em um algoritmo, as instruções são colocadas e ordenadas para que o seu objetivo seja alcançado.

Quando se cita "competências do século XXI" isso está relacionado a forma como aprendemos. Diante disso, o que se aprende não deve servir apenas como memorização, mas sim, precisa ter uma aplicação prática dentro desse fato. É nesse contexto que entra o Raciocínio Computacional que, de acordo com Wing (2006), "é um conjunto de habilidades intelectuais e de raciocínio que indicam como as pessoas interagem e aprendem a pensar por meio da linguagem computacional".

A partir do entendimento sobre Pensamento Computacional e dos conteúdos programáticos de Pré-Cálculo (e. g., Grupo GEN na Figura 1), verificou-se que ensinar as estratégias de Pensamento Computacional em conjunto com os conteúdos programáticos de Pré-Cálculo poderia mobilizar competências relacionadas ao raciocínio lógico e resolução de problemas nos estudantes calouros, que tem potencial de impacto positivo tanto no âmbito acadêmico quanto profissional e, consequentemente, minimizar as taxas de retenção e evasão no curso de Engenharia Civil do IFMS - Campus Aquidauana. 
Figura 1 - Conteúdos programáticos de pré-cálculo

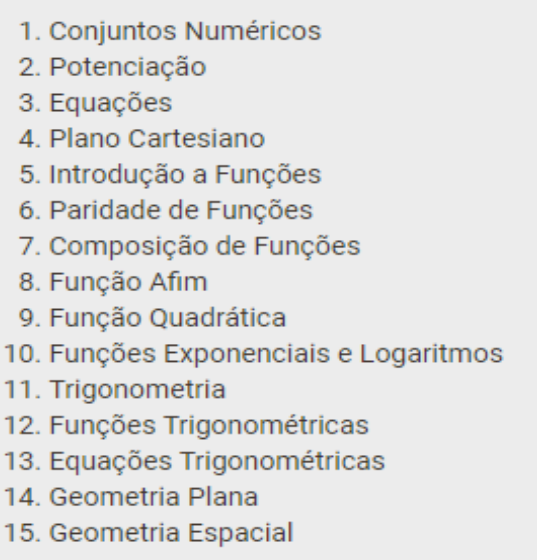

Fonte: Grupo GEN (2019)

Em relação ao processo de aplicação do Pensamento Computacional, buscou-se na literatura um modelo conceitual apropriado para tal. Na Figura 2 apresenta-se este modelo que visa, por meio da proposição de problemas contextualizados de engenharia, o enriquecimento e o aprofundamento dos conceitos matemáticos envolvidos pela definição de algoritmos e sua síntese por meio de simulação e análise.

Figura 2 - Modelo de Integração
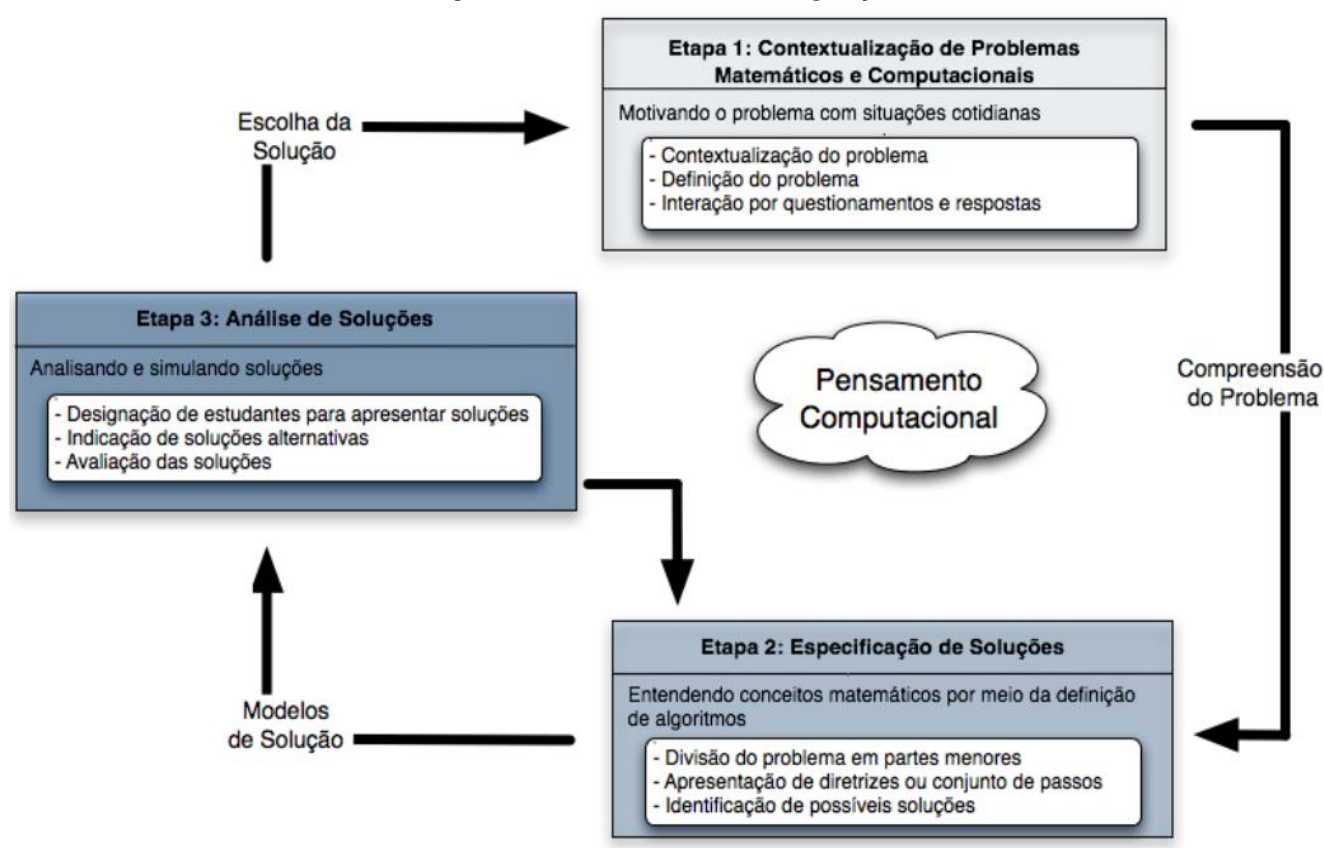

Fonte: Marques et. al. (2017)

Para estabelecer a intencionalidade pedagógica neste processo, utilizou-se a Taxonomia de Bloom (BLOOM, 1974) para a concepção de objetivos de aprendizagem. A estrutura desta taxonomia é pertinente para este trabalho pois fundamenta-se em processos cognitivos (i. e., Lembrar, Entender, Aplicar, Analisar, Avaliar e Criar) e tipos de conhecimento (i. e., Factual, Conceitual, Procedural e Metacognitivo).

A partir disso, elaborou-se os objetivos de aprendizagem com enfoque no Pensamento Computacional passando-se por todos os processos cognitivos e tipos de conhecimento, onde os Conteúdos Programáticos são utilizados para a proposição de problemas visando-se a resolução por meio do Pensamento Computacional. 
No Quadro 1 apresenta-se os resultados que culminaram na componente curricular.

Quadro 1 - Componente Curricular de Pensamento Computacional

Componente Curricular: Matemática com Pensamento Computacional

\section{Conteúdos Programáticos}

Operações com quatro operações. Frações. Mínimo Múltiplo Comum. Máximo divisor comum. Expressões numéricas. Potenciação. Radiciação. Racionalização de denominadores. Produtos notáveis. Fatoração em radicais. Equação do $1^{\circ}$ e $2^{\circ}$ grau. Números primos, fatores e divisores de um número, equações irracionais e biquadradas. Conjuntos. Introdução às funções. Função Afim. Função quadrática. Funções Exponenciais e Logarítmicas. Trigonometria. Funções trigonométricas. Equações trigonométricas.

\section{Objetivos de aprendizagem:}

Lembrar e entender métodos de decomposição do pensamento computacional interpretando os problemas e dividindo-os em vários outros pequenos problemas.

Reconhecer padrões em determinados problemas, resumindo os aspectos comuns do problema a ser resolvido.

Reconhecer elementos que tenham relevância em determinado problema, deixando de lado aqueles que não são importantes ao problema e reconhecendo a importância dos elementos selecionados para a solução do problema.

Entender como algoritmizar um problema, aplicando a heurística do pensamento computacional.

Resolver problemas usando da decomposição, do reconhecimento, da abstração e da algoritmização, atribuindo soluções coesas e organizadas, que obedecem a estrutura lógica do pensamento computacional.

Analisar problemas resolvidos através do pensamento computacional, discutindo entre pares e organizando estratégias para o aperfeiçoamento contínuo.

Avaliar soluções idealizadas por outros colegas utilizando pensamento computacional, sintetizando críticas construtivas e colaborativas.

Criar algoritmos utilizando o pensamento computacional em linguagem de programação, pseudocódigos e/ou fluxogramas, generalizando soluções para diferentes contextos.

\section{Bibliografia:}

\section{Básica}

JÚNIOR, Paulo Antonio Pascal; Pensamento Computacional e Tecnologias: reflexões sobre a educação no século XXI. Caxias do Sul, RS: Educs, 2020.

MEDEIROS, V. Z. Pré-cálculo, São Paulo: Cengage Learning, 2012.

ZEGARELLI, M. 1.001 Problemas de Matemática Básica e Pré-Álgebra Para Leigos. Rio de Janeiro: Alta Books, 2016.

\section{Complementar}

FRANKLIN, D. D.; BERT, K. W.; GREGORY, D. F.; DANIEL. K. Pré-cálculo. São Paulo: Addison Wesley, 2009.

IEZZI, Gelson; MURAKAMI, Carlos. Fundamentos de matemática elementar: conjuntos e funções. 8. ed. São Paulo: Atual, 2011. v. 1. 
IEZZI, Gelson; DOLCE, Osvaldo; MURAKAMI, Carlos. Fundamentos de matemática elementar: logaritmos. 9. ed. São Paulo: Atual, 2010. v. 2.

IEZZI, Gelson; MURAKAMI, Carlos. Fundamentos de matemática elementar: trigonometria. 8. ed. São Paulo: Atual, 2011. v. 3.

Fonte: elaborado pelos autores

\section{CONSIDERAÇÕES FINAIS}

Ao longo deste trabalho buscou-se esclarecer o que é (e o que não é) Pensamento Computacional tendo em vista a sua utilização como estratégia de ensino e aprendizagem em unidades curriculares relacionadas à matemática.

Cabe ressaltar que esta estratégia pode ser utilizada em qualquer unidade curricular pois o processo de "algoritmizar" não se reduz à linguagens de programação, mas sim, a um processo heurístico de resolução de problemas.

Tendo em vista os cursos de engenharia, a adoção do Pensamento Computacional tem potencial para transformar o contexto atual (aprendizagem baseada em memorização) em ambientes de aprendizagem ativa. Pois, devido à orientação para resolução de problemas concretos, cada estudante tem a oportunidade de ser protagonista da sua própria aprendizagem.

Por fim, sugere-se como trabalhos futuros a realização de avaliações de impacto desta proposta de componente curricular, visando-se verificar sua eficácia.

\section{REFERÊNCIAS}

BLOOM, B. S. Taxonomy of educational objectives: The classification of educational goals. Handbook 1-2. Longmans: McKay. 1974.

BOSCARIOLI, C. B. A modelagem matemática como metodologia para o desenvolvimento do pensamento computacional por meio da criação de objetos de aprendizagem. EDUCAÇÃO INTEGRAL POR MEIO DO PENSAMENTO COMPUTACIONAL, 1. ed. Curitiba: Appris Ltda. 2019. Disponível em:

https://institutoayrtonsenna.org.br/content/dam/institutoayrtonsenna/radar/estanteeducador/instituto-ayrton-senna-educacao-integral-por-meio-do-pensamento-computacional.pdf. Acesso em 23 dez. 2020.

Grupo GEN. Curso Online de Pré-Cálculo Interativo. 2019 Disponível em: https://www.grupogen.com.br/curso-online-pre-calculo-interativo\#summary. Acesso em: 07 de maio de 2021.

INSTITUTO AYRTON SENNA. Pensamento computacional e programação como ferramentas de aprendizagem. Disponível em: https://institutoayrtonsenna.org.br/pt-br/meu-educador-meuidolo/materialdeeducacao/pensamento-computacional-e-programacao-como-ferramentas-deaprendizagem.html. Acesso em 25 de fevereiro de 2021.

MACHADO, D; WARPECHOWSKI, M. COMPETÊNCIAS DO PENSAMENTO COMPUTACIONAL EM PRÁTICAS PEDAGÓGICAS DE PROFESSORES DA EDUCAÇÃO BÁSICA. IX MOSTRA INTEGRADA DE INICIAÇÃO CIENTÍFICA. Acesso em: $18 \mathrm{de}$ fevereiro de 2021. 
MARQUES, M; Cavalheiro, S; Foss, L; Bordini, A; Ávila, C. Uma Proposta para o Desenvolvimento do Pensamento Computacional Integrado ao Ensino de Matemática. Em Anais do XXVIII Simpósio Brasileiro de Informática na Educação (SBIE 2017). 2017, páginas 314-323.

\title{
MESTRE, P. USO DO PENSAMENTO COMPUTACIONAL COMO ESTRATÉGIA PARA
} RESOLUÇÃO DE PROBLEMAS MATEMÁTICOS. Dissertação submetida à Coordenação do Curso de Pós-Graduação em Ciência da Computação da Universidade Federal de Campina Grande Campus I. 2017. Campina Grande - Paraíba.

PIAGET, J. A Formação do Símbolo na criança. Editora: Livros técnicos e científicos. 1990.

Wing, M. J. "Computational Thinking". Communications of the ACM, CACM v. 49, n. 3, Março 2006, pp. 33-35.

\section{PICETTI, J. FORMAÇÃO CONTINUADA DE PROFESSORES: DA ABSTRAÇÃO}

REFLEXIONANTE Â TOMADA DE CONSCIÊNCIA. Tese apresentada ao programa de pósgraduação em educação. 2008. Porto Alegre.

\section{PICETTI, J. O PROCESSO DE ABSTRAÇÃO NA TRAJETÓRIA DA CONSTRUÇÃO DO CONHECIMENTO. Disponível em:}

http://maratavarespsictics.pbworks.com/w/file/fetch/74460728/Abstra\%C3\%A7\%C3\%A3o\%20Refl exionante.pdf. Acesso em: 19 de fevereiro de 2021.

\section{SANTOS, D. O DESENVOLVIMENTO DE HABILIDADES DO RACIOCÍNIO COMPUTACIONAL NA APRENDIZAGEM DE PROGRAMAÇÃO POR ESTUDANTES \\ INICIANTES. Trabalho de conclusão de curso. 2018. Salvador - Bahia.}

\author{
VEE, A. ENTENDENDO A PROGRAMAÇÃO DE COMPUTADORES COMO UM \\ LETRAMENTO. EDUCAÇÃO INTEGRAL POR MEIO DO PENSAMENTO \\ COMPUTACIONAL, 1. ed. Curitiba: Appris Ltda. 2019. Disponível em: \\ https://institutoayrtonsenna.org.br/content/dam/institutoayrtonsenna/radar/estante- \\ educador/instituto-ayrton-senna-educacao-integral-por-meio-do-pensamento-computacional.pdf. \\ Acesso em 17 fev. 2021.
}

\section{COMPUTATIONAL THINKING AND DIFFERENTIAL AND INTEGRAL CALCULUS: CURRICULAR COMPONENT PROPOSAL FOR IFMS - AQUIDAUANA CAMPUS}

\begin{abstract}
In the Higher Course in Civil Engineering at IFMS - Aquidauana Campus, a high failure rate was found, mainly in the curricular units related to Differential and Integral Calculus. After researching the literature, it was observed that the use of teaching-learning strategies that actively place the student in the process has generated better learning indicators. Thus, the objective of this paper was to design a proposal for a curricular component focused on students who are studying the curricular unit of Differential and Integral Calculus I, supporting the pedagogical praxis in Computational Thinking. The proposal was developed and is expected to verify its effectiveness in an impact evaluation in the future.
\end{abstract}

Keywords: Computational Literacy. Teaching-learning. Evasion. 